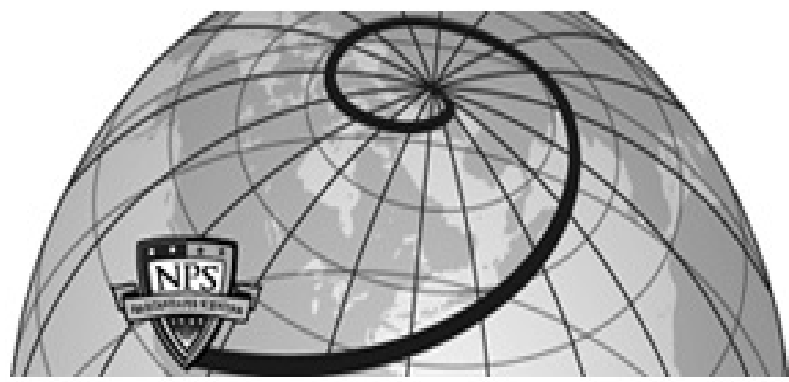

Calhoun: The NPS Institutional Archive DSpace Repository

\title{
Interconnecting Local Networks to Long-Distance Networks
}

Schneidewind, Norman F.

IEEE

Computer, September 1983

http://hdl.handle.net/10945/45145

This publication is a work of the U.S. Government as defined in Title 17, United States Code, Section 101. Copyright protection is not available for this work in the United States.

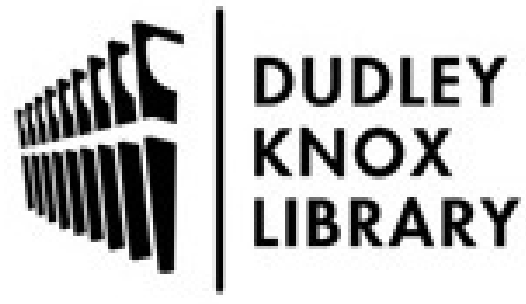

http://www.nps.edu/library
Calhoun is the Naval Postgraduate School's public access digital repository for research materials and institutional publications created by the NPS community. Calhoun is named for Professor of Mathematics Guy K. Calhoun, NPS's first appointed -- and published -- scholarly author.

Dudley Knox Library / Naval Postgraduate School 411 Dyer Road / 1 University Circle Monterey, California USA 93943 
The approaches to interconnection-network access, network services, and protocol functions-are related and overlap. User requirements and existing specifications determine which one the designer emphasizes.

\title{
Interconnecting Local Networks to Long-Distance Networks
}

\author{
Norman F. Schneidewind, Naval Postgraduate School
}

The demand for broad yet specific network services is an urgent one, and as both local and long-distance networks proliferate, the need for better connections between these disparate systems is becoming critical.

A local network is a data communications system that allows communication between a number of independent devices. These devices can be computers, terminals, mass storage devices, printers, plotters, or copying machines. ${ }^{1}$ The network may support a wide variety of applications, such as file editing and transfer, graphics, word processing, electronic mail, database management, and digital voice. Local networks are usually owned by a single organization and operated within a restricted geographical area, most often within a mile radius, at a moderate to high data rate such as 10 million bits per second. A long-distance network, on the other hand, is usually owned by a communications carrier and operated as a public utility for its subscribers, providing services such as voice, data, and video.

An emerging service of long-distance networks is providing the interconnection for local networks and other long-distance networks. Interconnection can provide

- local network to local network communication,

- local network to long-distance network communication, and

- long-distance network to long-distance network communication.

These interconnection possibilities, though exciting, inevitably present designers with the problem of resolving network incompatibilities. There are inherent differences in network characteristics as the result of the diversity of networks that exist to serve the various user communities. These differences have been accentuated by the failure of some networks to adopt standard protocols, by the variety of existing protocols and protocol standards, and by the wide range of incompatible options within existing protocol standards. In addition, designers are faced with a confusing mix of unregulated vendor products (e.g., IBM's System Network Architecture), local networks (e.g., Ethernet), regulated common carrier networks (e.g., Telenet), and newly deregulated communication services (e.g., American Bell).

\section{The changing network scene}

To set the stage for discussing the interconnection problem, let's review the evolution of computer networks to their current level of complexity.

Early connections. Before the advent of local networks, network services-supported by a single longdistance network-were viewed as shown in Figure 1. This view had the following major characteristics:

- terminal to computer communication,

- computer to computer communication, and

- terminal to terminal communication.

The orientation was one of data communication rather than networking. The long-distance network provided the terminal or remote-job-entry user with access to 
remote computers or terminals. This mode of operation was typical of, for example, earlier versions of Arpanet, Decnet, and SNA and of the services provided by data communications carriers such as AT\&T and MCI. The network provided access to hardware and software resources that were not available locally.

Emergence of networking. Rapid advances in technology, coupled with the development of local network architectures and protocols, led to the implementation of multiple mini/microcomputer-based workstations. The workstations are tied together by a physical communications medium and supported by protocols for network

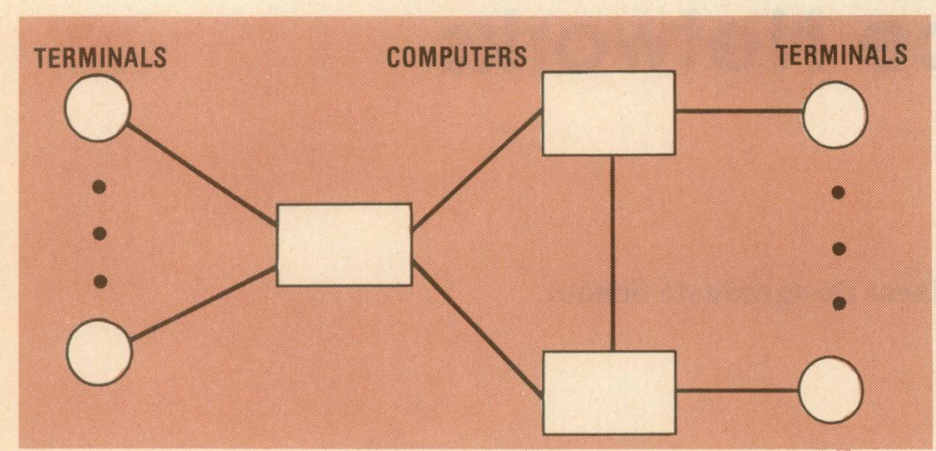

Figure 1. Previous view of computer networks. access and for message transmission and reception. Initially, the terminal was the focal point of activity, accessing hosts in a resource-sharing network such as Arpanet or using a long-distance communications network such as AT\&T for connection to a remote computer. Now, the emphasis has shifted to complete, self-contained local networks that access long-distance networks to communicate with other networks and, at the same time, provide communication among the local-network workstations. Thus, contemporary network services feature both inter-local and intra-local network communications (see Figure 2). As far as the long-distance network is concerned, the entire local network looks and functions like a single terminal. Indeed, this is an interconnection design goal.

\section{Interconnection issues}

Local and long-distance networks have significant differences that must be addressed when planning and designing networks consisting of one or more local networks interconnected by one or more long-distance networks.

The differences between local and long-distance networks, summarized in Table 1 , lead to major interconnection issues:

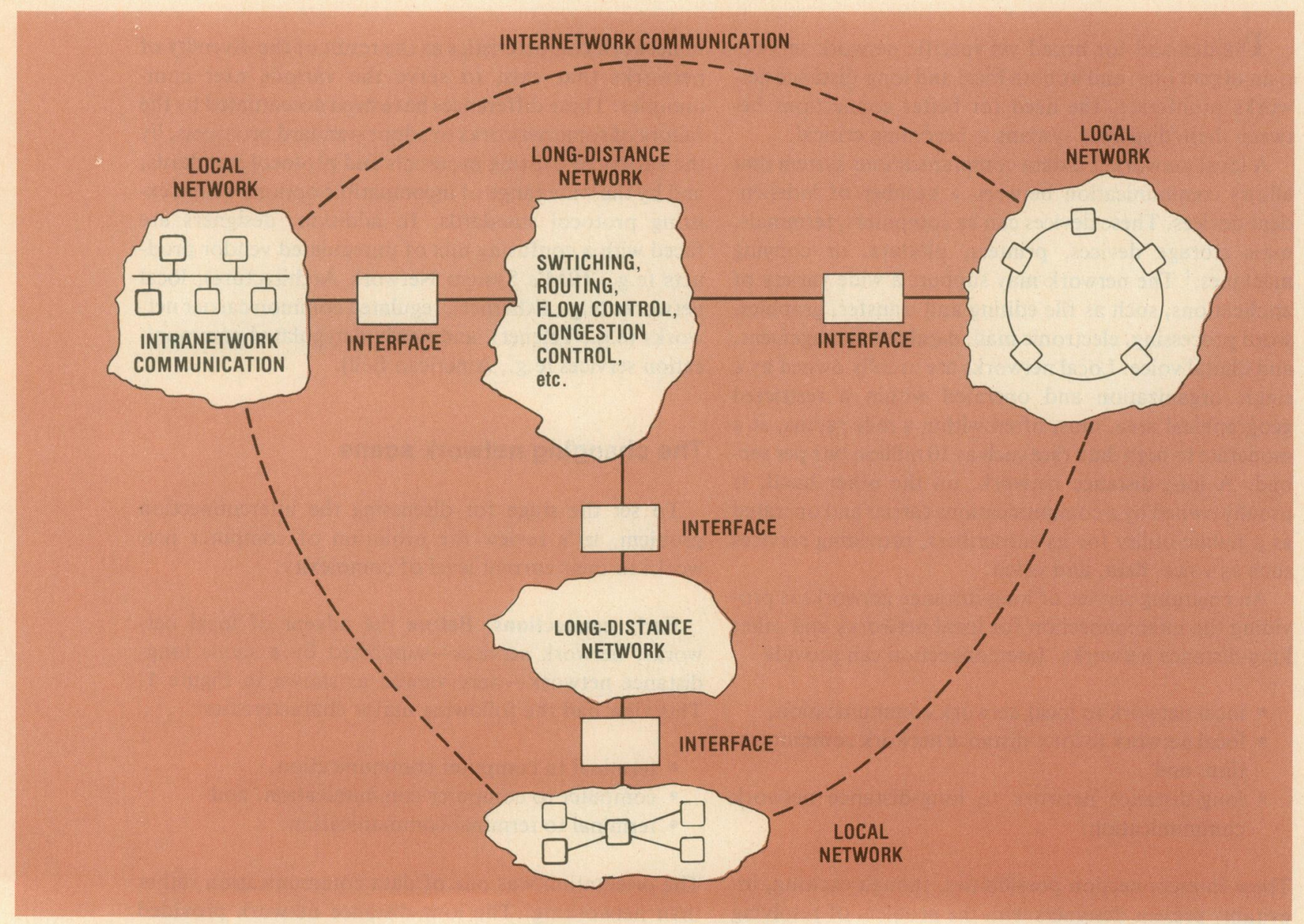

Figure 2. Contemporary view of computer networks. 
- How should a local network be physically attached to a long-distance network?

- How should one local network access and communicate with another local network?

- To what extent should protocol and network architecture standards used in one network be used in another network?

- How is the difference in bandwidth, as it affects acknowledgment handling and flow control, to be resolved?

- How is the difference in delay time, as it affects user response time, to be reconciled?

- How should network addressing capability be provided? (A single local network can, of course, have a simpler address structure than a long-distance network, but communication among multiple local networks is the most difficult addressing problem of all.)

Decisions regarding these interconnection issues must be made before, not after, the networks are implemented.

A variety of situations and factors can affect the type of interconnection provided. Sometimes the networks already exist and their specifications are known-for example, when an existing local network is connected to an existing long-distance network to communicate with other local networks. In other situations, however, one or more of the networks must still be specified. A common design problem involves developing specifications for nonexistent local networks that are to communicate via one or more existing long-distance networks.

Thus, the properties of the long-distance network(s) are usually given and part of the local network design problem involves the specification of the interface for connecting the two types of networks. For the designer, this situation presents both a challenge and an opportunity: a challenge because few user organizationsother than communications carriers or the Department of Defense-can control the characteristics designed into long-distance networks; an opportunity because the designer can influence the effectiveness of both intralocal and inter-local communication by the approach he uses to specify the network interface.

In designing the interface, the following important principle applies:

The more a local network is designed to increase the effectiveness of intra-local network communication, the more the cost of the interface to a long-distance network increases and the more the effectiveness of interlocal network communication decreases.

This principle is an outgrowth of the significant differences in the characteristics that distinguish local and long-distance networks (Table 1). These differences lead to a high-cost, complex interface if each type of network is tailored to the particular needs of its user communities. On the other hand, if the local network is designed to achieve compatibility with a long-distance network, the cost of the interface falls, but local-network throughput and response time suffer. The usual way of resolving this trade-off is to lean heavily in the direction of maximizing

local network effectiveness, at the expense of interface cost, because the interface represents a one-time cost while the local network must provide effective service for its users over the lifetime of the network.

\section{The ISO architecture}

A significant development in the standards area that exerts considerable influence on network design and intercommunication methods is the International Standards Organization model of architecture for open systems interconnection. $^{2}$ (See Figure 3.) The extent to

Table 1.

Comparison of local and long-distance network characteristics.

\begin{tabular}{lll}
\hline CHARACTERISTIC & LOCAL NETWORK & LONG-DISTANCE NETWORK \\
\hline Typical Bandwidth & $\begin{array}{l}10 \text { million bits per } \\
\text { second. }\end{array}$ & 56,000 bits per second. \\
Acknowledgment & $\begin{array}{l}\text { One message } \\
\text { acknowledged at a } \\
\text { time. }\end{array}$ & $\begin{array}{l}\text { N messages acknowledged at a } \\
\text { time. }\end{array}$ \\
$\begin{array}{l}\text { Message Size and } \\
\text { Format }\end{array}$ & $\begin{array}{l}\text { Small (simple } \\
\text { header). No need to } \\
\text { divide message into } \\
\text { packets. }\end{array}$ & $\begin{array}{l}\text { Large (complex header). Need } \\
\text { to divide message into packets. }\end{array}$ \\
\end{tabular}

Network Control

Minimum requirement due to small number of links and nodes and simple topology.

Flow/Congestion Control

Error Rate

Message Sequence and Delivery

Standard Architecture

Routing

Delay Time

Addressing
Minimum due to high bandwidth and simple topology.

Relatively low Operated in benign environment.

Minimum problem due to simple topology (e.g., bus or ring).

Usually only two or three bottom layers provided.

None required due to simple topology.

Small due to short distance and medium (e.g. coaxial cable)

Simple intra-network communication due to simple topology. Complex inter-network communication due to the use of long-distance network(s).
Extensive due to large number of nodes and links and complex topology

Extensive due to low bandwidth and complex topology.

Relatively high. Operated in noisy environment of telephone network.

Major problem due to complex topology (e.g., mesh)

Frequent use of all or many ISO layers

Major problem due to complex topology.

Large due to distance and medium (e.g., satellite).

Complex because of many nodes and links. 
which local and long-distance networks adhere to the ISO model has an important bearing on the nature and complexity of the interconnection. The use of many, or all, ISO layers is particularly important for long-distance networks because of their

- complex topology,

- wide geographical coverage,

- large number of nodes and links,

- extensive switching and routing,

- numerous points requiring flow control and congestion control,

- relatively long delays in end-to-end transmission, and

- long distance process-to-process communication requiring complex acknowledgment schemes.

Local networks, on the other hand, have much simpler characteristics and thus less need for the various ISO protocol services. Warner ${ }^{3}$ has suggested that forcing a local network design in total into the ISO mold could result in significant overhead in terms of (1) a large message size to accommodate multiple headers for the many ISO layers, (2) complicated and unnecessary message acknowledgment procedures, and (3) extraneous hardware and software to support the ISO transport and network layers. However, not using ISO in the local network risks loss of compatibility with ISO-based networks, one of

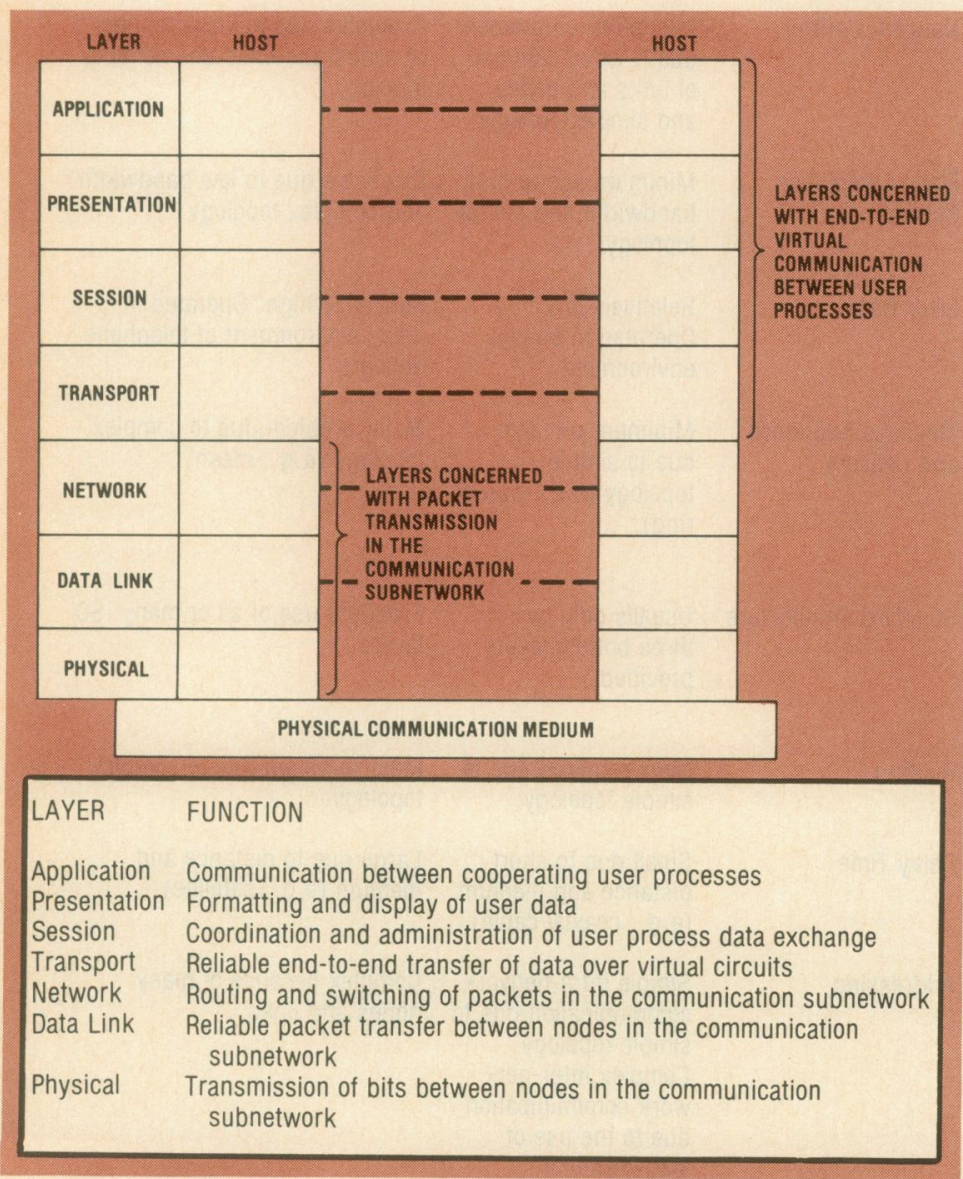

Figure 3. ISO model seven-layer architecture. which could be a long-distance network that must interface with the local network.

\section{Approaches for solving the interconnection problem}

The three basic approaches to interconnection-network access, network services, and protocol functions-are related and frequently overlap. Bear in mind that a combination of the approaches, described and compared below, may be necessary to interconnect diverse networks effectively.

Network access. The network access approach involves making electrical and physical connections of local networks and user communities via the longdistance network. The predominate considerations in this approach are ones of physical access and interfacing-for example, signal levels, pin connectors, cable length, and transmission medium. But it also emphasizes local-network compatibility with the long-distance network at the bottom three layers of the ISO model. All three layers are needed. Any network communication requires the data link layer for reliable internode communication, and a long-distance network requires the switching and routing functions of the network layer.

The main concern in this approach is the user's ability to access additional users and resources at a reasonable cost by the most convenient, feasible method. Whether or not the network services obtained as a result of the connection are ideal for the application is of secondary concern.

A number of situations could motivate user interest in the network access approach. Frequently, the motivation is convenience; the service needed-say, for example, an X.25-compatible network-might simply be readily available. Another possibility is that the user's objective is limited. He might not be interested in obtaining computer-based services such as database management, financial forecasting, or engineering computation; perhaps he only wants the services of a transmission medium for providing connectivity to other users. Then, the need is for a communications facility whose primary function is the transport of data. A data communications carrier is frequently the long-distance network that provides this level of service. A third possibility is that the user's terminal equipment is only compatible with certain networks, so the ability to provide interconnection at the lowest physical level outweighs other considerations.

Ordinarily, the network access approach is used when both the local networks and the long-distance network already exist. Since network characteristics cannot be created or modified, the user's options are limited, and he must be content with a low-cost network that is hardware-compatible with his local networks.

Network services. High-level user services can be obtained with the network services approach to interconnection. Some services, such as database management, could be related to the application and presentation layers of the ISO model; others, such as a high-level com- 
munication service like electronic mail, could be related to the transport and network layers.

Obviously, any interconnection approach involves connections between local networks and a long-distance network and thereby provides inter-local network access. However, in contrast to the first approach, the network services approach stresses obtaining specific network services for the user organization, and the details of physical and electrical interfacing, while important, become secondary issues. Necessarily, this approach emphasizes compatibility between local networks and a longdistance network at the higher level layers of the ISO model.

What conditions motivate a user to consider this approach? One situation is when the long-distance network provides a data management or processing service that is not available in the local networks. Often only a largescale network (e.g., Arpanet) has the host hardware and software capable of providing a desired service. A second situation arises when a high-level, inter-local network communication service is desired. For example, the user organization may want to tie its local networks together, or connect to other organizations' local networks to provide remote interactive processing involving session control and sequenced, error-free message delivery. Typically, this type of service would be obtained from a longdistance network that provides a virtual circuit service. Since the objective is to obtain a specific type of network service, naturally the long-distance network is an existing one. However, the local networks may or may not exist. If they do, protocol conversion will probably be required at the interface between the local networks and the longdistance network. If they don't exist and if ease of interfacing is a primary objective, the desired service may prompt the local network designer to attempt maximum compatibility with the layers and protocols of the longdistance network.

In general, a data communication network is appropriate for the network access approach, but a resourcesharing or value-added network is germane to the network services approach.

Protocol functions. The third method of achieving interconnection provides one set of protocol functions for the local network and another set for the long-distance network, where some functions, or corresponding network layers, are common to both networks. When crossing network boundaries, a transition or "conversion" is made between protocols. The boundary points are typically implemented in hardware with gateways (an interface between two networks) and front-end processors.

This method is used when the user's primary objective is to optimize local network communication, by using only those layers and protocols necessary for local network operation, while also providing communication between local networks via the long-distance network. Although the user will not be indifferent to physical access and network services, the dominant objective is to marry two diverse types of networks, which are inherently incompatible, and still retain the effectiveness of each. Protocol conversion is necessary to achieve this objective. The degree of conversion is a function of the dif- ferences between the layers and protocols in the two types of networks.

When would a user organization be able to capitalize on this approach? The most likely situation is when the local networks have not been implemented and the user has the opportunity to influence both intra-local network and inter-local network effectiveness by virtue of the protocol functions provided in the local networks; the long-distance network may or may not have been implemented (i.e., it may also be in the planning or development stage). Regardless of the status of the long-distance network, the user organization would, in most instances, have negligible influence over the design of the longdistance network due to the size and dominant position of the long-distance network organization.

\section{In some cases, one protocol can be translated into another. In others, protocols can be held in common among the communicating parties.}

This approach is seldom viable when the local networks exist; the likely degree of protocol conversion required, in terms of message format, message size, acknowledgment method, naming, addressing, error control, etc., would make it infeasible.

\section{Comparison of approaches}

As stated by Cerf and Kirstein, ${ }^{4}$ the common objective of all interconnection methods is to allow all subscribers a means of accessing a host on any of the interconnected networks. They go on to declare that achieving this objective requires that data produced at a source in one network be delivered and correctly interpreted at the destination(s) in another network. This reduces to providing interprocess communication across network boundaries. In some cases, it is enough to translate one protocol into another. In others, protocols can be held in common among the communicating parties.

The efficiency of achieving this objective depends on which approach is utilized. It is not necessary to implement all ISO layers in the local network to achieve effective intra-local network communication, nor is implementation necessary to connect to a long-distance network. However, the number, types, and characteristics of the layers utilized determine the efficiency of interlocal network communication (i.e., communication over the long-distance network).

The network access approach certainly achieves physical access to hosts, but the interconnection does not provide access to all the services available in the longdistance network. This approach does not fully achieve the requirement of delivering data from the source and having it correctly interpreted at the destination, because complete compatibility between local network protocols and long-distance network protocols may not be possible. For example, it might achieve a datagram service, which requires only the first three ISO layers, but might 
not be able to realize virtual circuit service, which requires use of the transport layer.

The network services approach does, on the other hand, completely satisfy the requirement regarding data delivery and interpretation, but not necessarily efficiently. In this approach, it may be necessary to adopt inefficient local network protocols to achieve compatiblity with the long-distance network. This is the case if the protocols are held in common among the communicating parties, where the "communicating parties" are the local network and the long-distance network.

Table 2.

Comparison of network interconnection approaches.

\begin{tabular}{|c|c|c|c|}
\hline APPROACH & CHARACTERISTIC & ADVANTAGES & DISADVANTAGES \\
\hline $\begin{array}{l}\text { Network } \\
\text { Access }\end{array}$ & $\begin{array}{l}\text { Achieves physical } \\
\text { access and protocol } \\
\text { compatibility in lower } \\
\text { layers of ISO model } \\
\text { (e.g., physical, data } \\
\text { link, and network } \\
\text { layers of X.25). }\end{array}$ & $\begin{array}{l}\text { Relatively } \\
\text { inexpensive. May } \\
\text { obtain compati- } \\
\text { bility with } \\
\text { international } \\
\text { standards. }\end{array}$ & $\begin{array}{l}\text { Only provides } \\
\text { compatibility with } \\
\text { lower layers of } \\
\text { network architecture. } \\
\text { Restricted to services } \\
\text { of network access } \\
\text { method. }\end{array}$ \\
\hline $\begin{array}{l}\text { Network } \\
\text { Services }\end{array}$ & $\begin{array}{l}\text { Obtains use of } \\
\text { specific types of } \\
\text { services (e.g., virtual } \\
\text { circuit service) to } \\
\text { satisfy user needs. }\end{array}$ & $\begin{array}{l}\text { The network } \\
\text { service is } \\
\text { matched to the } \\
\text { user need as } \\
\text { opposed to simply } \\
\text { obtaining physical } \\
\text { access to a } \\
\text { network. Reduces } \\
\text { software develop- } \\
\text { ment expense. }\end{array}$ & $\begin{array}{l}\text { Restricted to using } \\
\text { the same (perhaps } \\
\text { inefficient) protocols } \\
\text { in all interconnected } \\
\text { networks. May be } \\
\text { restricted to using a } \\
\text { single type of } \\
\text { service. }\end{array}$ \\
\hline $\begin{array}{l}\text { Protocol } \\
\text { Functions }\end{array}$ & $\begin{array}{l}\text { Matches number and } \\
\text { types of protocols to } \\
\text { each type of network. }\end{array}$ & $\begin{array}{l}\text { Uses only those } \\
\text { protocols that are } \\
\text { necessary in each } \\
\text { type of network. } \\
\text { High perfor- } \\
\text { mance. Low } \\
\text { overhead. }\end{array}$ & $\begin{array}{l}\text { Expensive software } \\
\text { development. } \\
\text { Complex network } \\
\text { interface. }\end{array}$ \\
\hline
\end{tabular}

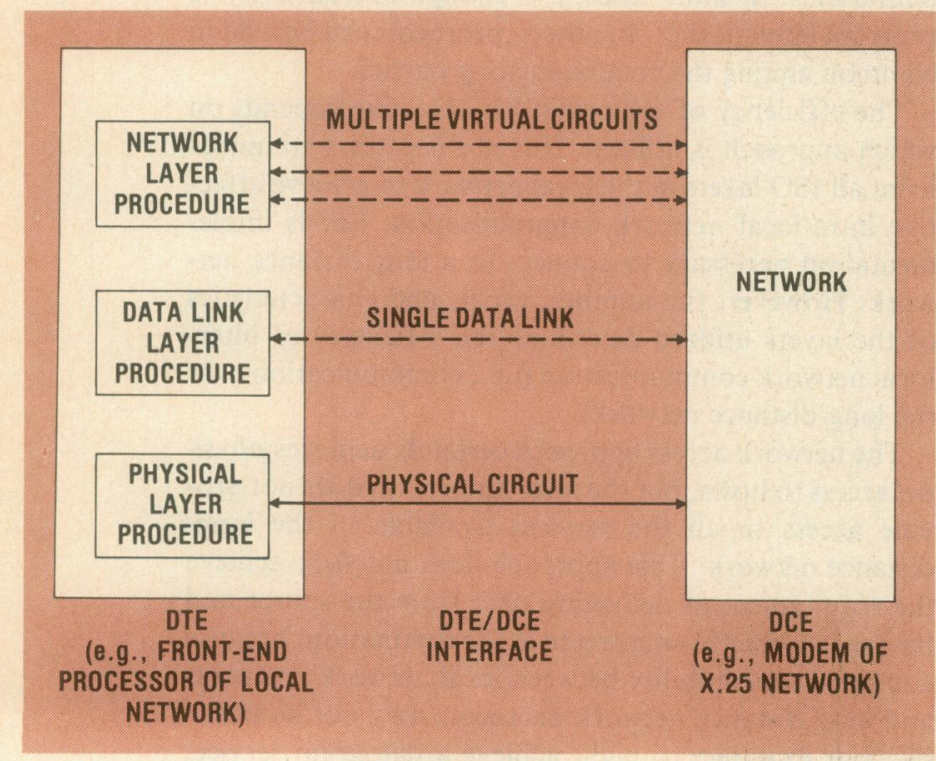

Figure 4. Structure of the X.25 interface (adapted from Reference 5).
The protocol functions approach solves the problem of local-network communications efficiency, but at high hardware and software costs. Its use of the protocol translation technique necessitates a complex network interface.

In general, no matter which approach is utilized, the owner of the local network is responsible for designing the interface and arranging the connection with the owner of the long-distance network, using the specifications of that network as a guide. This work will take place over an extended period of time, and involves development, design, implementation, and maintenance. The three approaches are summarized and compared in Table 2.

\section{Examples of interconnection approaches}

Network access. Rybczynski ${ }^{5}$ provides an example of the network access approach to interconnection vis-a-vis the X.25 interface standard. X.25 is a standard deviceindependent interface between X.25-compatible packet networks and user devices operating in the packet mode. The interface point occurs between the user's data terminal equipment, or DTE, and the data communications equipment, or DCE, of an X.25 packet switching network (e.g., GTE-Telenet). Although the DTE is often thought of as an individual terminal or host, it could just as well be a front-end processor of a local network. The DCE could be a modem associated with the communication channel of an X.25 packet-switching node. Multiple local networks connected in this fashion would provide inter-local network communication and the services offered by the X.25 long-distance network. The interface between the DTE and DCE occurs at the first three layers of the ISO model, as shown in Figure 4.

The characteristics of these layers* are as follows:

\section{Physical layer}

- full duplex,

- point-to-point, and

- synchronous.

\section{Data link layer}

- data link control procedures compatible with the high-level data, link control (HDLC) protocol,

- single data links,

- data transfer,

- link synchronization,

- detection of and recovery from errors, and

- reporting of errors to a higher layer.

\section{Network layer}

- packetizing of user and control data,

- network addressing, and

- multiple virtual circuits.

From a practical standpoint the user organization must provide the appropriate hardware and software in

"The X.25 designations for the ISO network and data link layers are "packet" and "frame," respectively; X.25 also uses "level" rather than the ISO term "layer." The ISO terminology is used here to maintain consistency. 
its network access unit (e.g., a front-end processor) in the physical, data link, and network layers to be compatible with X.25. This means, for example, providing software to implement HDLC in the data link layer and an RS232-C hardware interface in the physical layer.

This approach to interconnection emphasizes physical access and the use of the long-distance network as a communications medium for the exchange of messages between local networks. The interconnected local networks must adopt the standards of the long-distance network and install the lower level protocols (e.g., physical, data link, and network layers of X.25) of the long-distance network. This will be beneficial or detrimental for the operation of the local networks, depending on whether the protocol characteristics (acknowledgement, addressing, etc.) enhance or degrade the performance of the local networks. Since this approach only addresses the lower level protocols, the user must provide the upper layer protocols. Also, since network performance is primarily a function of upper-layer characteristics, achievable performance will be uncertain. The major advantage of this approach is its low cost, since the required protocol software exists for many computers.

DDN, the defense data network, illustrates the limitations of the network access approach, specifically with regard to X.25. Although using X.25 is not currently a DDN requirement, it may be added to permit communication with network nodes in the NATO community. ${ }^{6}$ The switching nodes used in the DDN (BBN Model C.30s) are designed to be compatible with X.25 in all three layers. However, layer 3 of X.25, the network layer, is not compatible with the layer-3 host-IMP protocol used to support communication between a local network and the DDN. Without the installation of a gateway (i.e., the protocol functions approach), it will not be possible for hosts in the DDN to communicate with hosts in NATO. Thus, although X.25 protocol software exists in DDN, offering the potential for a low-cost interconnection, no local network can presently connect to DDN using X.25 protocols; it would not be compatible with all the DDN protocols. Achieving this compatibility would require a protocol converter for translation between the host-IMP and X.25 network layer protocols.

Network services. Another example using X.25 illustrates the relationship between network access and network services. In this example, the real significance of achieving X.25 network access compatibility is to obtain X.25 network services such as switched virtual circuits, permanent virtual circuits, and datagrams.

SVCS and PVCs. Switched virtual circuits, or SVCs, provide a temporary logical circuit between two host processes. A call request action by the process that wants to open a connection is required. An SVC will compete for the use of available network resources, and possibly experience delay in establishing the circuit, but has the advantage of using these resources efficiently. On the other hand, a permanent virtual circuit, or PVC, guarantees network access to the user, obviating the call request procedure, and provides a data path between a fixed pair of network endpoints. The SVC and PVC services are analogous to the switched network and leased lines services offered by data communications carriers.

Datagrams. A datagram service provides transmission of packets in a network where each packet is independent of other packets. There is no guarantee of sequenced delivery of packets constituting a message, nor guarantee of any type of delivery, for that matter. A datagram service is fast and uses network resources efficiently-for example, the destination node can pass a message to a host without waiting to reassemble all of its packets or tieing up valuable buffer space for this purpose. If the user desires guaranteed sequenced delivery, it must be provided by the transport layer residing above the network layer, which is charged with datagram delivery.

\section{It is one thing for users to tolerate an occasional lost packet but quite different to forego sequential delivery.}

A datagram service is useful in certain applications where no higher layers are necessary to complete the job of message delivery and where all the data to carry out a function can be contained in a single packet-for example, transaction processing such as a file update, where each update represents an independent action, and narrative message transmission, where each message can be considered an independent entity. In some applications-digitized voice, for example-a datagram service is used primarily because of its speed.

Most practical applications of datagram services require the transport layer to sequence datagrams for delivery to a host process. It is one thing for all the data in a packet to be self-contained (e.g., a Fortran compile command) or to tolerate an occasional lost packet (e.g., digitized voice) but quite different, from the standpoint of the user's operation, to forego sequential delivery; the user wants a Fortran compile done next or the parts of a voice conversation to reach the listener in sequence. (Subsequent sections show how the Department of Defense uses the datagram concept in combination with upper layer functions to provide the desired network services.)

$D o D D D N$. An application where X.25 takes on great importance is the Department of Defense's Defense Data Network. ${ }^{6}$ As related by Corrigan, ${ }^{7}$ planners of DDN were confronted with the following situation:

(1) For the most part, vendor protocols are incompatible across different manufacturers' equipment.

(2) However, the DDN must allow for various vendor protocols because many DoD user communities, using a great variety of vendor hardware and software, must be accommodated in the DDN.

(3) Although the Arpanet architecture and protocols, on which the DDN is based, provide many of the capabilities desired for the DDN, they are not completely compatible with the various vendor protocols. 
(4) X.25 has been adopted by manufacturers but has not been implemented in the DDN.

Cerf and Lyons ${ }^{8}$ point out that, although X.25 is a network access standard for connecting to packetswitched networks, it has the following deficiencies relative to its use in the DDN:

(1) Although datagram service is part of the standard, it has not been implemented in any network. The datagram mode is essential to real-time military applications, such as tactical operations and packet voice.

(2) The availability of only virtual circuit service in $X .25$, with its required sequencing, introduces intolerable delays in certain real-time applications. In many applications, total data integrity is not essential, but minimum delay is mandatory. Certain military applications require a broadcast mode, which is not compatible with sequenced delivery in a virtual circuit.

\section{Given the diversity of network characteristics, the network services strategy may be good for inter-local network operations but not for intra-local network performance.}

Thus, the DoD is faced with a dilemma: compatibility with X.25 is important because many vendors and communications carriers have adopted it, but X.25 does not fully meet DoD's requirements. The DoD's response to this problem is fourfold ${ }^{8}$ :

(1) The Internet protocol ${ }^{9}$ has been developed for the DDN and is now operational on Arpanet. IP provides a datagram service for those applications which require fast, nonsequenced delivery and addressing and routing capabilities for transmission of datagrams across multiple networks.

(2) The Transmission Control Protocol ${ }^{9}$ has been developed for the DDN and is now operational on the Arpanet. Using the services of IP, and the additional capabilities of sequenced delivery, flow control, and end-to-end acknowledgment, TCP provides a virtual circuit service for applications that require interactive terminal to remote host processing.

(3) As a long-range goal, DDN compatibility with $\mathrm{X} .25$ is planned.

(4) The Defense Communications Agency is working with the international standards bodies (e.g., ISO and CCITT) to promote acceptance of TCP/IP in the civilian community and to urge these bodies to give more weight to military requirements, such as real-time response, security, precedence message handling, and survivability, in their standards efforts.

From the above, it can be seen that achieving interconnection among diverse networks can very likely involve a combination of approaches: network access (e.g., X.25), network services (e.g., datagram and virtual circuit), and political and standards activity (e.g., ISO).

The important factor in the network services approach to interconnection is obtaining services available in the long-distance network for the user organization's local networks. Given the diversity of network characteristics, this strategy may be good for inter-local network operations but not for intra-local network performance. Such penalties as high software overhead, due to unneeded flow control, routing, and addressing capabilities, are especially prevalent if all layers of the long-distance network are incorporated into local network communication operations. Even inter-local network efficiency can be poor if service is limited to one type (e.g., fixedrouting virtual circuit service). However, this approach has the significant advantage of providing clean interfaces at the network boundaries, due to the use of the same protocols in all networks. The implication of this is reduced software development costs compared to methods that tailor the protocols to the characteristics of each interconnected network.

Protocol functions. An example illustrating the protocol functions approach is the Navy's Stock Point Logistics Integrated Communications Environment system. The SPLICE concept is being developed as a result of growing demands for automated data processing at Navy stock points and inventory control points. SPLICE is designed to augment the existing Navy stock point and inventory control point ADP facilities, which support the Uniform Automated Data Processing System-Stock Points. The hardware for the UADPS-SP consists of Burroughs medium-size systems. At present there are 20 new application systems being developed that require considerable interactive and computer communication support. These new application systems will utilize minicomputers capable of supporting foreground interactive and computer communication requirements.

Two major objectives led to the development of SPLICE: first, the increased need for the use of interactive database processing to replace the current batchoriented system; second, the need to standardize the current multitude of interfaces. To reduce total system cost, SPLICE will be developed using a standard set of minicomputer hardware and software. Standardization is particularly important because SPLICE will be implemented at some 60 geographical locations, each having a different mix of application and terminal requirements. The SPLICE processors will be co-located with the host Burroughs system at each Navy stock point and with Burroughs and Univac systems at the inventory control points. A "foreground-background" concept will be implemented with SPLICE minicomputers, which will serve as front-end processors for the Burroughs systems via a local area network interface. The Burroughs computers will provide background processing functions for large file processing and report generation.

The SPLICE project ${ }^{10}$ has the following characteristics:

- intra-local area network communication, 
- inter-LAN communication over the long-distance DDN,

- conflicting requirements-LAN vs. long-distance network-for communication,

- mandated communications protocols, required for compatibility purposes, that have little relevance to LAN communication,

- local and remote interactive and batch processing, and

- off-loading of certain processing (e.g., database management) from mainframes to minicomputers.

The network configuration (Figure 5) shows the integration of the three interconnection methods-network access, network services, and protocol functions. Network access is achieved via the hardware of the frontend processor in conjunction with the access line and the host-IMP protocol, a network access protocol used in the Arpanet and adapted to the DDN. Basic network services consist of a virtual circuit service for interactive processing and other operations that require reliable, sequenced end-to-end message delivery, plus a datagram service for operations which involve the transmission of independent messages (e.g., the sending of a message in the electronic mail system). The datagram service, implemented in the internet protocol (IP) and operating in the network layer, ${ }^{*}$ supports the transmission control protocol (TCP), operating in the transport layer, to provide a virtual circuit service. The datagram service is used to obtain efficient bandwidth utilization and flexibile message routing. This flexibility is achieved because the messages can be transmitted as independent packets without being constrained to follow a single path and to follow one another in sequence. The TCP sequences the messages

*Some authors classify this as an eighth layer-the internet layersituated between the transport and network layers. and provides reliable end-to-end delivery. The protocol functions approach provides only those protocols in the local area network that are needed to support this type of communications environment. Due to the great differences between local and long-distance network characteristics, protocol requirements differ significantly. This point is illustrated in Table 3 , which delineates differences in the utilization of ISO layers and protocols in LAN and DDN communication.

Sending and receiving messages on the DDN will use all seven layers of the ISO model, as shown in Table 3 . The LAN has no need for the services normally provided by the transport and network layers, because routing, switching, and traditional flow control and congestion control services are unnecessary. The presentation layer, implemented in the terminal management module, will accept data from the application process and convert it to LAN format. Conversely, it will accept messages in LAN format and convert them to the appropriate application process format.

Table 3.

Use of ISO layers in LAN design.

\begin{tabular}{|c|c|c|}
\hline LAYER & $\begin{array}{l}\text { LAN COMMUNICATION } \\
\text { PROTOCOL/MODULE }\end{array}$ & DDN COMMUNICATION \\
\hline Application & Application Process Modules & Same as for LAN \\
\hline Presentation & Terminal Management & Terminal Management \\
\hline Session & Session Services & Session Services \\
\hline Transport & -- & TCP \\
\hline Network & -- & IP \\
\hline $\begin{array}{l}\text { Data Link } \\
\text { Physical }\end{array}$ & $\begin{array}{l}\text { Local Communications } \\
\text { Local Communications }\end{array}$ & $\begin{array}{l}\text { Specified by the DDN } \\
\text { (Various Protocols) }\end{array}$ \\
\hline
\end{tabular}

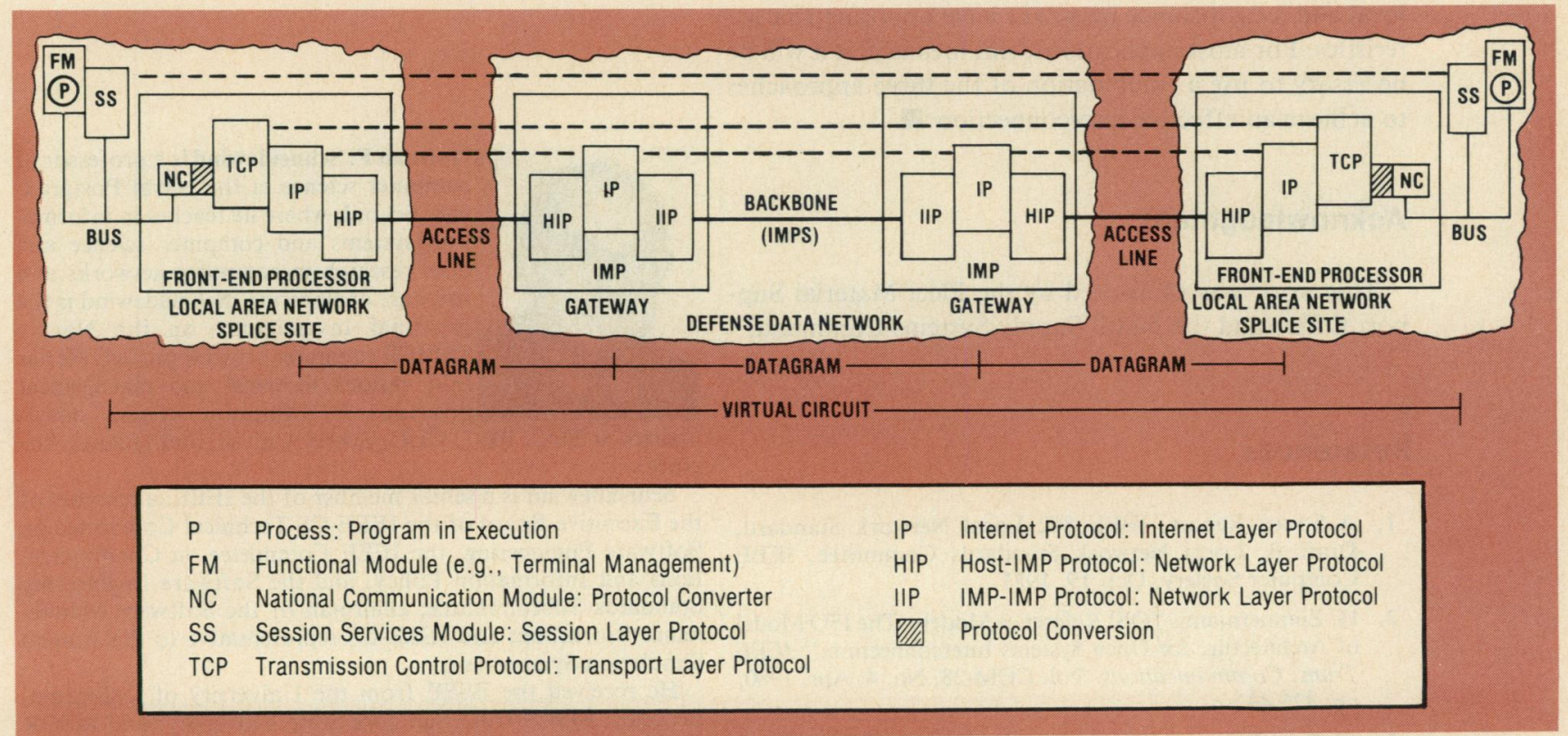

Figure 5. Relationship between local area networks and the Defense Data Network. 
To simplify the LAN design, the following message formats are used:

(1) TCP format will be provided to the DDN by the NC module (see Figure 5) whenever communication on the DDN is necessary. A much simpler format will be used for intra-LAN communication.

(2) End-to-end virtual circuit connections and breaking of complete messages into fragments, services normally provided by the transport layer, will be implemented in each of the LAN modules. "Endto-end" in this context refers to the logical communication linkage between two modules separated by a relatively short distance; in some cases the two modules could be in the same hardware unit.

To maximize compatibility and minimize software development, the protocols in the two networks are selected to match as closely as possible, consistent with satisfying the requirements of vastly different communications environments (e.g., routing in the DDN and no routing in the LAN).

The protocol functions approach is used where the inter-local network services and performance provided by the long-distance network (e.g., combination of virtual circuit and datagram services) are satisfactory. It is also used where optimization of intra-local network performance is desired. This is accomplished by using only those layers and protocols that are compatible with and can take advantage of the characteristics of local networks (e.g., single message acknowledgment made possible by high bandwidth). Of course, the user organization must be willing to pay the relatively high software cost of this tailored approach.

It is apparent from the discussion and examples that users may have diverse networking requirements and that local and long-distance networks have opposing characteristics. For most applications, this means that it will be necessary to use a combination of the three approaches to achieve an effective interconnection.

\section{Acknowledgment}

This work was sponsored by the Fleet Material Support Office and the Naval Supply Systems Command.

\section{References}

1. A Status Report, IEEE 802 Local Network Standard, Draft B, Local Network Standards Committee, IEEE Computer Society, Oct. 19, 1981.

2. H. Zimmermann, “OSI Reference Model-The ISO Model of Architecture for Open Systems Interconnection," IEEE Trans. Communications, Vol. COM-28, No. 4, Apr. 1980, pp. $425-432$.

3. Clifford Warner, "Connecting Local Networks to Long Haul Networks; Issues in Protocol Design," Proc. Fifth
Conf. Local Computer Networks, IEEE Computer Society, Oct. 1980 , pp. 71-76.

4. V. G. Cerf and P. T. Kirstein, "Issues in Packet-Network Interconnection," Proc. IEEE, Vol. 66, No. 11, Nov. 1978, pp. 1386-1408.

5. A. Rybczynski, "X.25 Interface and End-End Virtual Circuit Service Characteristics," IEEE Trans. Communications, Vol. COM-28, No. 4, Apr. 1980, pp. 500-510.

6. Defense Data Network Program Plan, Defense Communications Agency, Jan. 1982, revised May 1982.

7. Michael L. Corrigan, "Defense Data Network Protocols," Conf. Record, EASCON 82, 15th Annual Electronics and Aerospace Systems Conf., Washington, DC, Sept. 1982, pp. 131-135.

8. V. G. Cerf and R. E. Lyons, "Military Requirements for Packet-Switched Networks and Their Implications for Protocol Standardization," op cit., pp. 119-129.

9. Jonathan B. Postel, "Internetwork Protocol Approaches," IEEE Trans. Communications, Vol. COM-28, No. 4, Apr. 1980, pp. 604-611.

10. N. Schneidewind, "Functional Approach to the Design of a Local Network: A Naval Logistics System Example," Compcon Spring 83 Digest of Papers, IEEE Computer Society, Mar. 1983, pp. 197-202.

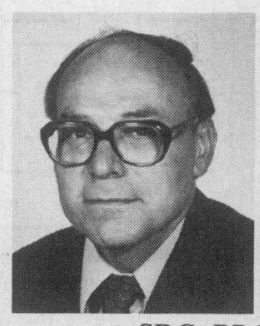

Norman F. Schneidewind is a professor of computer science at the Naval Postgraduate School, where he teaches in information systems and computer science and does research in computer networks and software engineering. Schneidewind is the principal investigator on the Navy's SPLICE computer network project. He has held various technical and management positions in computer system development at SDC, PRC, CUC, Sperry Univac, and Hughes Aircraft.

Schneidewind is a senior member of the IEEE; a member of the Executive Board of the IEEE-CS Technical Committee on Software Engineering, the IEEE Committee on Communications and Information Policy, and the Software Engineering Standards Subcommittee; chairman of the Software Maintenance Workshop; and IEEE-CS representative to the Annual Simulation Symposium.

He received the BSEE from the University of California, Berkeley; MSCS from San Jose State University; and MSOR (Engr), MBA, and DBA from the University of Southern California; and holds the Certificate in Data Processing. 